Service social

\title{
La pauvreté : mieux la connaître et mieux la comprendre
}

\section{Hector Ouellet et Suzie Robichaud}

Volume 44, numéro 3, 1995

Pauvreté

URI : https://id.erudit.org/iderudit/706703ar

DOI : https://doi.org/10.7202/706703ar

Aller au sommaire du numéro

Éditeur(s)

École de service social de l'Université Laval

ISSN

1708-1734 (numérique)

Découvrir la revue

Citer ce document

Ouellet, H. \& Robichaud, S. (1995). La pauvreté : mieux la connaître et mieux la comprendre. Service social, 44(3), 3-6. https://doi.org/10.7202/706703ar d'utilisation que vous pouvez consulter en ligne.

https://apropos.erudit.org/fr/usagers/politique-dutilisation/ 


\section{AVANT.PROPOS}

\section{La pauvreté : mieux la connaître et mieux la comprendre}

L'année 1996 aura été, à la demande des Nations Unies, I'année internationale pour l'élimination de la pauvreté. Cette décision aura pu surprendre par son idéalisme. Elle nous semble néanmoins porteuse de quatre messages majeurs. Le premier et le plus simple de ces messages tient au rappel de l'importance de la question de la pauvreté et de l'omniprésence de celle-ci, même dans les sociétés dites avancées : en singularisant ainsi cette question, en la remettant à l'ordre du jour, l'ONU lui aura redonné une légitimité nouvelle.

Par ailleurs, s'il apparaît approprié de décider qu'une année sera "l'année internationale pour l'élimination de la pauvreté ", c'est donc essentiellement qu'on estime qu'on peut intervenir sur la pauvreté et que, contrairement à ce que trop de discours politiques, économistes et moraux laissent entendre (quand ils ne le disent pas explicitement), celle-ci n'est pas une fatalité: on peut agir sur elle, elle est le résultat $d^{\prime}$ un processus identifiable modifiable et réversible. La pauvreté est un effet de l'activité de l'homme; sa solution peut donc tout aussi bien venir de lui. C'est là le deuxième message que nous lisons dans la décision de l'ONU.

Au Canada, la réticence généralisée (sinon le refus explicite) de nos gouvernements à revoir notre fiscalité, à interdire le recours aux paradis fiscaux, à contraindre l'utilisation des échappatoires fiscales, 
les interventions répétées et systématiques de la Banque du Canada, au cours des dernières années, en vue de ralentir délibérément l'économie, et donc de faire croître le chômage, nous apparaissent toutes découler du recours à la thèse dite de l'effet de cascade (trickling down effect). Les tenants de cette thèse font valoir "qu'avant de distribuer la richesse, il faut d'abord la créer ", laissant ainsi entendre qu'une fois créée, la richesse se redistribuera bien d'elle-même.

Selon cette même thèse, si nous "libérons " I'économie, si nous cessons de contraindre les "investisseurs », quitte à rendre "momentanément " plus difficiles les conditions de vie de pans entiers de la population, le développement et la richesse ultimement créés se répercuteront immanquablement et d'eux-mêmes sur le bien-être de toute la collectivité et des individus qui la composent. Implicitement, la pauvreté est ainsi vue comme une "condition» de développement.

Or, si stratégiquement utile que puisse être, parfois, la défense de cette thèse, celle-ci nous semble loin d'avoir démontré sa véracité. II nous semble de plus que, par sa décision, l'ONU ne la reconnaît pas comme valide: plutôt qu'à se distribuer d'elle-même, la richesse tend au contraire à se concentrer et des mesures massives doivent être entreprises si l'on veut voir la pauvreté régresser. C'est là le troisième message qui nous est livré par la décision de I'ONU de faire de l'année 1996 "l'année internationale pour l'élimination de la pauvreté ".

Finalement, et $c^{\prime}$ est le quatrième message que nous lisons dans la décision de l'ONU, celle-ci nous semble proposer une thèse située aux antipodes de celle qui voit la pauvreté comme condition de développement: elle y verrait au contraire plutôt un frein, un handicap au développement.

Par sa prise de position, I'ONU nous place, par ailleurs, devant les limites de nos réflexions et analyses en ce qui a trait à la pauvreté. Et ces limites apparaissent dans toutes les étapes de la réflexion. Nous comprenons encore très mal les éléments essentiels qui constituent ce phénomène. La validité de nos instruments de mesure demeure encore très faible; ceux-ci ne nous permettent pas d'analyser le phénomène de façon satisfaisante et, surtout, ils sont largement impuissants à le présenter dans sa complexité. Nous en connaissons encore trop peu sur la pauvreté vécue par des populations spécifiques et sur les handicaps ainsi provoqués sur les potentialités de développement de ces mêmes populations. Finalement, nous savons encore très mal comment il nous serait possible $d^{\prime}$ agir sur ce phénomène.

$C^{\prime}$ est avec la volonté de combler au moins une partie de ces lacunes que le présent numéro thématique a été conçu. On y trouvera des documents qui, chacun de leur côté, tenteront d'apporter un 
éclairage sur chacune des dimensions que nous venons d'esquisser: la compréhension du phénomène, sa mesure, sa signification pour certaines populations particulièrement affectées, de même que des hypothèses $d^{\prime}$ interventions.

Ce numéro thématique débute par une discussion sur la notion de pauvreté et son évolution. Après un bref rappel historique, Lucie Mercier présente une synthèse de l'évolution des approches et des définitions les plus courantes sur la pauvreté, des façons d'identifier les pauvres et des moyens utilisés pour circonscrire et mesurer le phénomène. Cet article conduit à situer, dans cette évolution, les notions plus récemment apparues pour décrire le phénomène de la pauvreté, qui sont celles de précarité, de fragilité et de désaffiliation sociale. La perspective sera ici surtout analytique.

Suit une contribution majeure de nature plus méthodologique. Simon Langlois et François Gardes y cherchent à renouveler la discussion sur la mesure du phénomène de la pauvreté. Après avoir passé en revue et critiqué les mesures habituellement utilisées pour le décrire, ces auteurs proposent une nouvelle mesure de la pauvreté appelée indice synthétique de pauvreté-richesse (ISPR). Cet indice fait appel à trois dimensions centrales de la pauvreté: le revenu, les dépenses totales et la privation des ménages. Par cet indice, les auteurs visent moins à fournir un moyen de "compter les pauvres» qu'à proposer un nouvel instrument d'analyse qui permet de meilleures comparaisons interrégionales et internationales que celles rendues possibles par les instruments habituels. À titre d'illustration, cet indice est appliqué au Québec. Son utilisation permet de conclure que si la pauvreté demeure élevée au Québec et qu'elle est en croissance dans certains groupes de la population, l'écart avec l'Ontario serait moindre que celui présenté par les mesures habituelles de la pauvreté.

$L^{\prime}$ article de Madeleine Gauthier, Andrée Roberge et Louise Saint-Laurent se concentre sur les questions et perspectives de recherche concernant la pauvreté chez les jeunes: il s'agit d'une population chez qui les taux de pauvreté sont particulièrement élevés. Les auteures signalent la méconnaissance de cette problématique telle que vécue par cette population. On comprendra mieux, dès lors, la difficulté et la fragilité des interventions auprès des jeunes pauvres. En incitant à refaire la réflexion sur le phénomène de la pauvreté, ces auteures donnent à leur article une perspective critique.

À partir d'une perspective interventionniste, Louis Favreau et Lucie Fréchette centrent leur réflexion sur la pauvreté urbaine. Voyant dans la pauvreté essentiellement un phénomène $d^{\prime}$ exclusion sociale faisant appel à une vision duale de la société ( $d$ 'un côté les inclus, 
d'un autre les exclus), les auteurs présentent la notion d'empowerment comme approche de solution à la pauvreté. Plus concrètement, ils présentent les Corporations de développement communautaire (CDC) et les Corporations de développement économique communautaire (CDEC) comme justement des moyens de favoriser l'empowerment des populations exclues et donc de lutter contre la pauvreté.

À travers une analyse de l'évolution de l'aide alimentaire dans la région de Québec, Joël Rouffignat invite le lecteur à lutter contre la pauvreté, mais aussi (et peut-être d'abord) à revoir les conceptions habituellement acceptées de la pauvreté et à reprendre l'analyse de la question en privilégiant la "recherche conscientisante» comme moyen particulièrement adapté à la compréhension intime du phénomène. Ce n'est que par une meilleure compréhension que nous serons mieux en mesure $d^{\prime}$ intervenir.

Nous ne pouvions produire ce numéro thématique sur la pauvreté sans présenter la discussion sur la réforme de l'aide sociale actuellement en cours et, plus précisément, sans exposer les travaux récents du Comité externe de la réforme de la sécurité du revenu. Ces travaux ont conduit à la préparation de deux rapports, I'un signé par Camil Bouchard, Vivian Labrie et Alain Noël (1996) et intitulé Chacun sa part (ministère de l'Emploi et de la Solidarité, Gouvernement du Québec); I'autre signé par Pierre Fortin et Francine Séguin (1996) intitulé Pour un régime équitable axé sur l'emploi (ministère de I'Emploi et de la Solidarité, Gouvernement du Québec).

Nous avons ainsi demandé à Camil Bouchard et Pierre Fortin de nous présenter, pour ce numéro de la revue, un résumé de chacun de leurs rapports. On trouvera donc ici le texte de M. Bouchard, La réforme de l'aide sociale. Enjeux et propositions concernant la lutte à la pauvreté, qui résume les propositions essentielles du rapport dont il est le coauteur. Nous regrettons par ailleurs que M. Fortin n'ait pu accéder pour le moment à notre demande.

Ce numéro thématique sur la pauvreté se termine par un texte de Jean-Pierre Bélanger et Gilles Desrosiers, que I'on trouvera dans la section "Commentaires et documents". En présentant le développement des organismes communautaires comme hypothèse de solution à la pauvreté, ce document complète particulièrement bien ce numéro de la revue Service social.

En bref, donc, plusieurs articles, construits selon des perspectives différentes mais qui tous, nous le croyons, contribuent à renouveler la réflexion sur la pauvreté. 